\title{
OPTICAL COHERENCE TOMOGRAPHY ANGIOGRAPHY TO DETECT AND MONITOR NONEXUDATIVE NEOVASCULARIZATION IN AGE-RELATED MACULAR DEGENERATION
}

\author{
Manuel A P Vilela \\ Medical School, Federal University of Health Sciences of Porto Alegre, RS, Brazil.
}

\begin{abstract}
OCT angiography has improved the ability to identify silent or nonexudative neovessels in age-related macular degeneration. This promotes a rapid advancement in the diagnosis and in the necessity to expand our management decisions. This context is presented and the biomarkers are discussed.

Keywords: Optical coherence tomography; Optical coherence tomography angiography; age-related macular degeneration

Cite This Article: VILELA, manuel a p. OPTICAL COHERENCE TOMOGRAPHY ANGIOGRAPHY TO DETECT AND MONITOR NONEXUDATIVE NEOVASCULARIZATION IN AGE-RELATED MACULAR DEGENERATION. International Journal of Retina, [S.I.], v. 4, n. 1, p. 72, feb. 2021. ISSN 2614-8536. Available at: <https://www.ijretina.com/index.php/ijretina/article/view/128>. Date accessed: 22 feb. 2021. doi: https://doi.org/10.35479/ijretina.2021.vol004.iss001.128.
\end{abstract}

*Correspondence to:

Manuel A P Vilela,

Federal University of Health

Sciences of Porto Alegre

Porto Alegre, Brazil

mapvilela@gmail.com

\section{INTRODUCTION}

Age-related macular
degeneration (AMD) has
many new contexts.
These are necessary given
that prevalence is

projected to rise in the next 20 years to as much as $75 \%$, or 175 million patients. The current standard diagnostic tools routinely combine clinical retinal examination with dye-based angiographies and structural ocular coherence tomography (OCT). Using these resources we identified the site of the disease and monitored its activity. Another important aspect to consider is related to neovessel resilience in AMD even with prolonged anti-VEGF therapy, given that the MARINA Study found that these neovessels do not disappear. ${ }^{1,2}$

Further relevant information came from different clinical spectra. Nowadays the Gass-Freund classification is used and helps to provide a prognosis. Basically, there are 3 subtypes of neovascularization: (type 1) sub-retinal pigment epithelium (RPE) or occult; (type 2) subretinal or classic or well-defined; retinal angiomatous proliferation or deep retinal neovascular complex (type 3). ${ }^{3,4}$

Recently the use of OCT angiography (OCTA) has revealed the presence of nonexudative neovessels undetected by any other resource and the necessity to establish the best form of case management. But this situation is not new at all. Almost 45 years ago histological studies showed sub-RPE neovessels in $11 \%$ of eyes that had previously been diagnosed as having intermediate AMD (iAMD). Probably the blockage of fluorescein caused by the RPE, the low flow inside these vessels, and the absence of exudation prevent the image from being captured by the resources used. ${ }^{5}$ With the use of indocyanine green angiography (ICGA) which has better access under the RPE this situation was detected in around $11 \%$ of the cases with $\mathrm{A} A M D$ and with negative intravenous fluorescein angiography (IVFA). 
ICGA was able to show the different evolution (2.6 $x$ more likely to exudate) or impaired visual acuity without exudation. Lesions tended to enlarge over time, causing visual distortions even in the absence of exudation.

The objective of this paper is to review the current information concerning the detection of neovessels in IAMD and the biomarkers that help in the evaluation of their activity

\section{CLINICAL DETECTION AND BIOMARKERS}

During many years the risks for AMD cases are related to drusen size. After 10 years almost $15 \%$ of patients with medium drusen in both eyes will develop late AMD, and for fellow eye cases almost $60 \%$ or more will develop late $A M D$, depending on the number of risk factors. ${ }^{7}$

The introduction of OCT has pointed out indirect intraretinal spots, external limiting membrane discontinuity, ellipsoid breaks, and growth of pigment epithelium detachment are related to the presence of these neovessels. Some modifications in the structure of the retina are strong indicators, but the vessels are unseen. ${ }^{8,9}$

OCTA has given us new definitions. Nonexudative macular neovascularization in AMD (nneAMD) is defined as type 1 lesion detected with dye-based angiography or OCTA that is not associated with clinical or structural OCT evidence of exudation for at least 6 months. There is a 30\% chance of exudation in that period ${ }^{10-13}$ Another definition, "nonexudative detachment of the neurosensory retina" (NEDNR) characterizes the presence of subretinal fluid (SRF) in iAMD that was not associated with any detectable macular

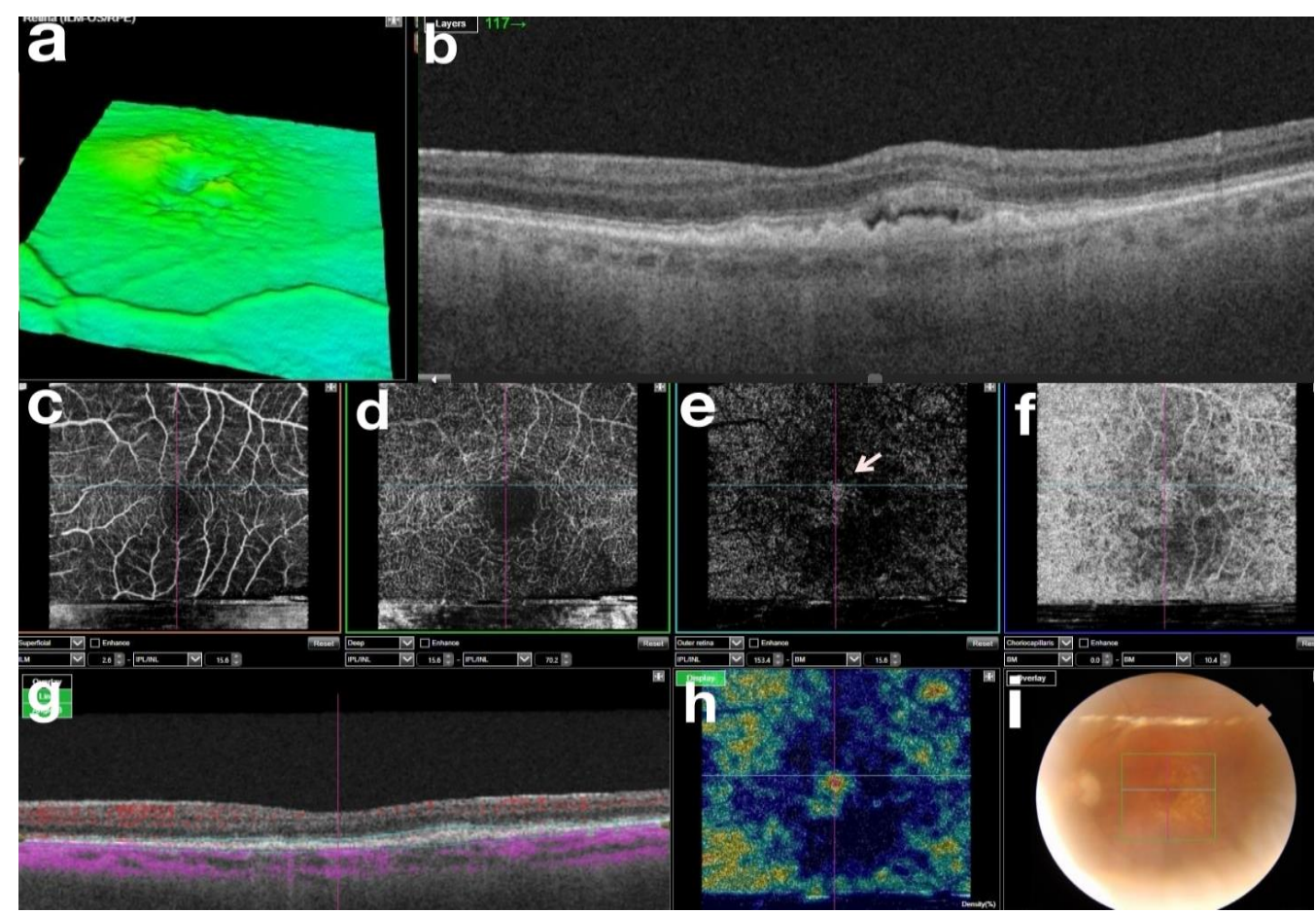

Figure 1. NONEXUDATIVE NEOVASCULARIZATION IN AGE-RELATED MACULAR

DEGENERATION - SS-OCT (DRI - OCT1 - TRITON Plus Topcon, Tokyo, Japan).

Drusen are shown in 3D reconstruction (a) and color photograph (i). The OCT-B mode (b) and OCT$B$ flow analysis ( $g$ ) show the presence of fluid. The OCT-A segmentation (c,d,e,f) identify a small neovascular tuft (arrow). The flow vessel density $(h)$ localizes the tiny neovascularization.

signs of the presence of these nonexudative or quiescent or silent neovessels. Findings like neovascularization using current state-of-the-art imaging, including SS-OCTA. ${ }^{14}$ (figure 1 and 2) 
Prevalence of nneAMD ranges from 5.5-34.0\% (mean 21.1\%) depending on the series. Studies have used different methodologies related to sample size, inclusion, exclusion, stage of AMD, equipment, manual or automatic segmentation. This heterogeneity explains this variation in the prevalence rates. But this average and the quality of observation is better than the routine combinations to achieve the same objective. ${ }^{11,14-17}$ OCTA sensitivity is near to $80 \%$, and probably a
A further consideration is that blood flow persists in all cases with nneAMD even after prolonged therapy. This is strong evidence that the treatment is not always necessary. Moreover, remission is not free from recurrence. Remission lasts longer in the case of eyes with low neovessels density and these cases must be closely monitored. Remission is caused by the RPE surrounding the tufts or regression of immature vessels. Lower vessel density is associated with subretinal fibrosis and

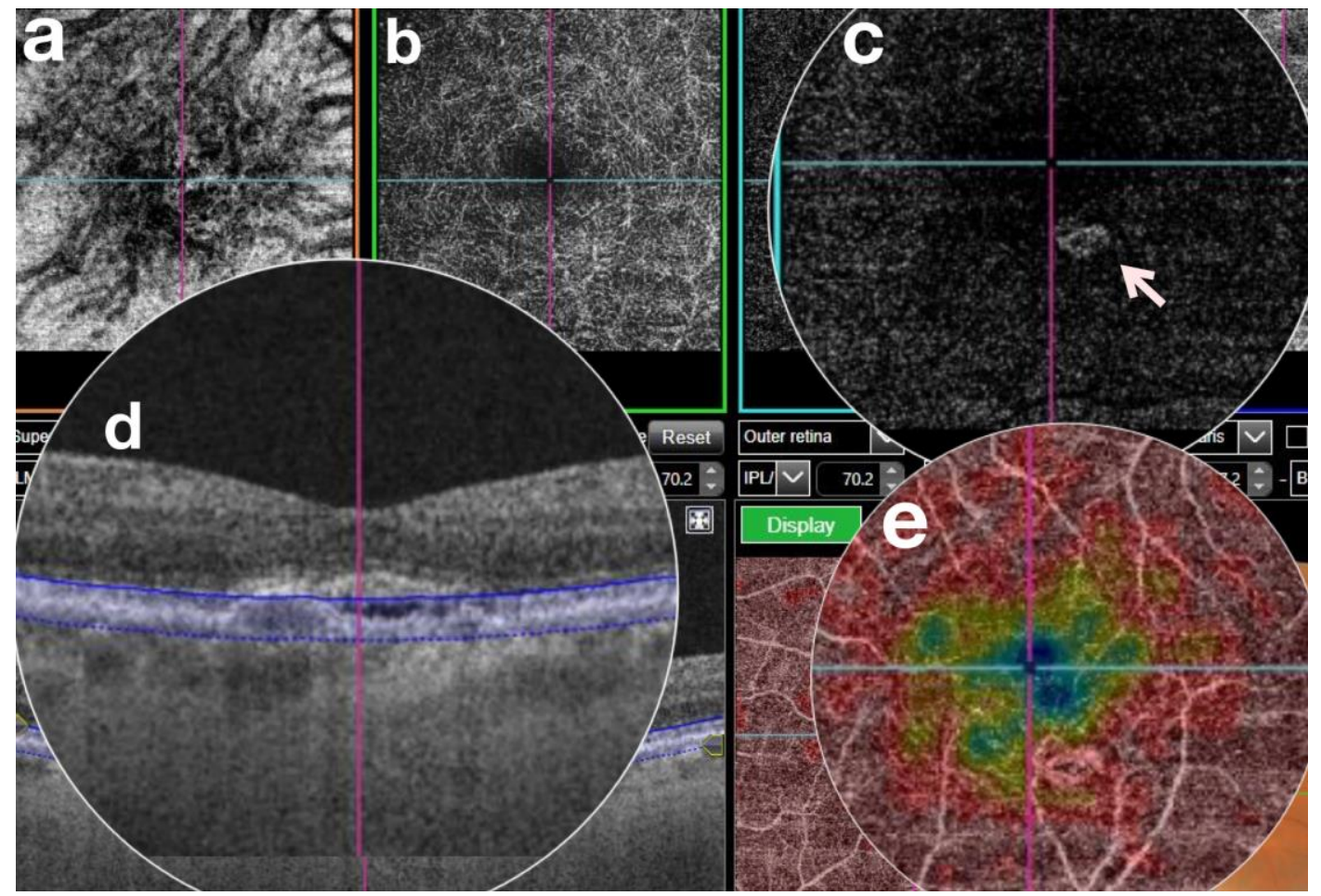

Figure 2. NONEXUDATIVE NEOVASCULARIZATION IN AGE-RELATED MACULAR

DEGENERATION - SS-OCT (DRI - OCT1 - TRITON Plus Topcon, Tokyo, Japan).

OCT-A segmentation $(a, b, c)$ identify the small neovascularization (arrow). OCT-B (d) shows the drusen and fluid. In the vessel density map (e), the flow void zones are localized around the fovea.

little bit lower if only type 1 is considered. This means that an important number of cases with nneAMD are not diagnosed with this technique. Specificity could be as high as $100 \% .^{18-21}$ Another relevant limitation is related to natural history. In nneAMD, exact evolution is poorly understood. The relative risk for exudation could be 15.2 times more compared with AMD without CMNV. In a year, 21.1$30 \%$ of these cases (versus $3.6 \%$ of normal cases) will develop active neovascularization. ${ }^{16}$ low VA. ${ }^{18-23}$ One of the best explanations about this is called the "vascular abnormalization theory". ${ }^{24}$

The artifacts produced during OCTA image acquisition (segmentation errors, projections, shadowing) are great challenges to be overcome and can interfere with precise evaluation. It is also important to highlight that most of the studies of this subject excluded patients with AMD associated with high myopia, cataracts, and diabetic retinopathy. These exclusions limit the conclusions that can be drawn. ${ }^{13,25,26}$ 
OCTA has made some considerable contributions to understand the pathogenesis. Standing out among them is the demonstration of dark areas and the flow void points also around the neovascular lesions. These halos should be correlated with low flow signs in structural OCTA. The situation reflects zones of bad perfusion (real ones or tiny vessels with sluggish flow) and is assumed to be related to primary AMD pathogenesis. Drusen can also co-localize choriocapillary impairment. ${ }^{13,27-29}$ Another important contribution derives from the analysis of the retinal and optic disc vessels in these cases. Both superficial and deep retinal and optic disc plexus are altered among patients with not-late AMD. The involvement of optic disc (deep and radial plexus) vessel density was also found to be reduced in the fellow eyes, and this could be a predictive sign. So choroidal thickness, vessel density and/or tortuosity could express vascular perfusion integrity, whereas vessel dispersion and rarefaction could measure damage to the retinal vessels. ${ }^{29-31}$ Observation of these modifications may improve sensitivity in detecting subclinical alterations occurring in these patients and lead to earlier therapeutic interventions.

Detection of biomarkers is of paramount importance for all patients with nneAMD. Both OCT-B and OCT-A have become the gold standard methods for detecting nnAMD. The combination of three of the situations can identify signs related to a worse prognosis: (1) a well-defined vessel with a lacy-wheel or sea-fan pattern (rather than long filamentous linear vessels); (2) great number and branching of small capillaries (rather than a few, large vessels); (3) detection of anastomoses and loops; (4) presence of peripheral arcade at the vessel terminus (not a "dead tree" appearance); (5) presence of a perilesional hypointense halo at the level of the choriocapillaris; (6) hyperreflective drusenoid lesions with flow evidence 8, 13,17, 27,32-34.
In conclusion, nneAMD is easier to detect with OCTA than with other resources, although not in all cases, neovessels uses not disappear completely. The main limitations are related to artifacts, and biomarkers could help to decide the moment to begin treatment.

\section{REFERENCES}

1. Wong WL, Su X, Li X, et al. Global prevalence of age-related macular degeneration and disease burden projection for 2020 and 2040: a systematic review and meta-analysis. Lancet Glob Health. 2014;2:e106-e116.

2. Rosenfeld PJ, Brown DM, Heier JS, et al; MARINA Study Group. N Engl J Med 2006; 355:14191431.

3. Gass JDM. Stereoscopic atlas of macular diseases: diagnosis and treatment. 4 ed. St Louis, Mosby CV, 1997.

4. Freund KB, Ho IV, Barbazetto IA, et al. Type 3 neovascularization: the expanded spectrum of retinal angiomatous proliferation. Retina 2008;28:201-211.

5. Sarks SH. Ageing and degeneration in the macular region: a clinico-pathological study. $\mathrm{Br} J$ Ophthalmol 1976; 60(5):324-341

6. Hanutsaha $P$, Guyer DR, Yannuzzi LA, et al. Indocyanine-green videoangiography of drusen as a possible predictive indicator of exudative maculopathy. Ophthalmology 1998; 105:16321636.

7. Chew EY, Clemons TE, Agrón E, et al; AgeRelated Eye Disease Study Research Group. JAMA Ophthalmol. 2014 Mar;132(3):272-7.

8. Schmidt-Erfurth $U$, Waldstein SM. A paradigm shift in imaging biomarkers in neovascular agerelated macular degeneration. Progress in Retinal and Eye Research 2015;50:1-24 
9. Fragiotta $S$, Rossi $T$, Cutini $A$, et al. Predictive factors for development of neovascular agerelated macular degeneration. Retina 2018; 38:245-252.

10.Querques G, Srour M, Massamba N, et al. Functional characterization and multimodal imaging of treatment-naive "quiescent"choroidal neovascularization. Invest Ophthalmol Vis Sci. 2013;54(10):6886-92.

11.Palejwala NV, Jia Y, Gao SS, e al. Detection of nonexudative choroidal neovascularization in age-related macular degeneration with optical coherence tomography angiography. Retina 2015; 35(11):2204-11.

12.Carnevali A, Cicinelli MV, Capuano V, et al. Optical coherence tomography angiography: a useful tool for diagnosis of treatment-naive quiescent choroidal neovascularization. Am J Ophthalmol 2016; 169:189-198.

13.Borrelli E, Sarraf D, Freund KB, Sadda SR. OCT angiography and evaluation of the choroid and choroidal vascular disorders. Progress in Retinal and Eye Research 2018; 67:30-55.

14.Lek JJ, Caruso E, Baglin EK, et al. Interpretation of subretinal fluid using OCT on intermediate age-related macular degeneration. Et al.Ophthalmology Retina 2018; 2(8):792-802.

15.Roisman L, Zhang Q, Wang R,Gregori G, et al. Optical coherence tomography angiography of asymptomatic neovascularization in intermediate age-related macular degeneration. Ophthalmology 2016;123:1309-1319.

16.Dias JRO, Zhang Q, Garcia JMB et al. Natural history of subclinical neovascularization in nonexudative age-related macular degeneration using swept-source OCT angiography. Ophthalmology 2018; 125:255266.

17.Or C, Heier Js, Boyer D, et al. Vascularized drusen: a cross-sectional study. Int J Retin Vitr 2019; 5:36
18.Sadda S. Defining the role of OCT angiography in clinical practice. Ophthalmolol Retina 2017;1(4):261-262.

19.Faridi A, Jia Y, Gao SS, et al. Sensitivity ans specificity of OCT angiography to detect choroidal neovascularization. Ophthalmolol Retina 2017; 1(4):294-303.

20.Cicinelli MV, Cavalleri $M$, Consorte $A C$, et al. Swept-source and spectral domain optical coherence tomography versus dye angiography in the measurement fo type 1 neovascularization. Retina 2019 Jan 14. doi: 10.1097/IAE.0000000000002452. [Epub ahead of print].

21.Lupidi M, Cerquaglia A, Chhablani J, et al.Optical coherence tomography angiography in agerelated macular degeneration: the game changer. Eur J Ophthalmol 2018;28(4):349-357.

22.Dhrami-Gavazi E, Balaratnasingam C, Lee W, Freund KB. Type 1 neovascularization may confer resistance to geographic atrophy amongst eyes treated for neovascular agerelated macular degeneration. Int J Retin Vitr 2015;1:15.

23.Ichiyama $Y$, Sawada $T$, Ito $Y$, et al. optical coherence tomography angiography reveals blood flow in choroidal neovascular membrane in remission phase of neovascular age-related macular degeneration. Retina 2017;37:724-730

24.Spaide RF. Optical coherence tomography angiography signs pf vascular abnormalization with antiangiogenic theraphy for choroidal neovascularization. Am J Ophthalmol 2015; 160(1): 6-16.

25.Lindner $M$, Fang PP, Steinberg JS, et al. OCT angiography-based detection and quantification of the neovascular network in exudative AMD. Invest Ophthamol Vis Sci 2016; 57:6342-48. 
26.Inoue $M$, Jung JJ, Balaratnasingam $C$, et al, for the COFT1 Study Group. A comparison between optical coherence tomography angiography and fluorescein angiography for the imaging of type 1 neovascularization..Invest Ophthalmol Vis Sci 2016; 57:OCT314-323

27.Kuehlewein L, Bansal $M$, Lenis $T L$, et al.Optical coherence tomography angiography of type 1 neovascularization in age-related macular degeneration. Am J Ophthalmol 2015; 160(4):739-48.

28.Wirth MA, Freiberg F, Pfau $M$, et al. Optical coherence tomography angiography in agerelated macular degeneration: persistence of vascular network in quiescent choroidal neovascularization.. Acta Ophthalmol 2017;95(4):428-430.

29.Toto L, Borrelli E, Antonio LD, et al. Retinal vascular plexuses changes in dry age-related macular degeneration, evaluated by means of optical coherence tomography angiography. Retina 2016;36(8):1566-72.

30.Gattoussi S, Cougnard-Gregoire A, Korobelnik $J F$, et al. Choroidal thickness, vascular factors, and age-related macular degeneration, The Alienor Study. Alienor Study. Retina 2019;39:3443.
31.Arrigo A, Aragona E, Capone $L$, et al. Advanced optical coherence tomography angiography of age-related macular degeneration complicated by onset of unilateral choroidal neovascularization.. Am J Ophthalmol 2018; 195:233-42.

32.Al-Sheikh $\mathrm{M}$, lafe NA, Phasukkijwatana $\mathrm{N}$, et al. Biomarkers of neovascular activity in agerelated macular degeneration using optical coherence tomography angiography. . Retina 2018;38:220-30.

33.Coscas F, Cabral D, Pereira T, et al. Quantitative optical coherence tomography angiography biomarkers for neovascular age-related macular degeneration in remission. PLOS ONE 2018;13(10):e0205513.

34.MENTES J, YILDIRIM S. OPTICAL COHERENCE TOMOGRAPHY CHARACTERISTICS OF QUIESCENT TYPE 1 NEOVASCULARIZATION IN EYES WITH NONEXUDATIVE AGE-RELATED MACULAR DEGENERATION. TURK J OPHTHALMOL 2019;49:84-88

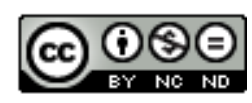

This work licensed under Creative Commons Attribution 